\title{
Referenzwerte pädiatrische Endokrinologie: Schilddrüsenvolumen
}

\section{Martin Wabitsch}

In Tab. 1 sind die alters- und geschlechtsabhängigen Schilddrüsenvolumina von Kindern und Jugendlichen aufgeführt.

Tab. 1 Sonografisch bestimmte alters- und geschlechtsabhängige Schilddrüsenvolumina bei Berliner Kindern. (Nach Liesenkötter et al. 1997)

\begin{tabular}{|c|c|c|c|c|}
\hline \multirow[b]{2}{*}{ Alter } & \multicolumn{3}{|c|}{ Schilddrüsenvolumen (ml) Mittelwert \pm SD } & \\
\hline & Jungen & Mädchen & Gesamt & \\
\hline$<6$ & $1,2 \pm 1,0$ & $1,5 \pm 1,4$ & $1,4 \pm 0,6$ & \\
\hline $6-7$ & $2,1 \pm 0,8$ & $2,5 \pm 1,0$ & $2,3 \pm 0,9$ & $3,6 \%$ \\
\hline $7-8$ & $2,3 \pm 0,8$ & $2,5 \pm 1,0$ & $2,4 \pm 0,9$ & \\
\hline $8-9$ & $2,4 \pm 0,7$ & $2,5 \pm 1,0$ & $2,4 \pm 0,9$ & \\
\hline $9-10$ & $3,0 \pm 0,7$ & $2,7 \pm 1,1$ & $2,8 \pm 0,9$ & $4,5 \%$ \\
\hline $10-11$ & $3,8 \pm 1,5$ & $4,2 \pm 1,4$ & $4,0 \pm 1,7$ & \\
\hline $11-12$ & $3,9 \pm 1,5$ & $4,4 \pm 2,1$ & $4,2 \pm 1,8$ & \\
\hline $12-13$ & $4,1 \pm 1,4$ & $4,9 \pm 2,3$ & $4,5 \pm 1,9$ & $4,9 \%$ \\
\hline $13-14$ & $4,4 \pm 1,9$ & $4,6 \pm 2,7$ & $4,5 \pm 2,3$ & \\
\hline $14-15$ & $4,4 \pm 1,4$ & $4,9 \pm 2,3$ & $4,6 \pm 2,7$ & \\
\hline
\end{tabular}

$S D$ Standardabweichung

\section{Literatur}

Liesenkötter KP, Kiebler A, Stach B, Willgerodt H, Grüters A (1997) Small thyroid volumes and normal iodine excretion in Berlin schoolchildren indicate full normalization of iodine supply. Exp Clin Endocrinol Diabetes 105(Suppl 4):46-50

\section{Wabitsch ( $\bowtie)$}

Sektion Pädiatrische Endokrinologie und Diabetologie Hormonzentrum für Kinder und Jugendliche, Klinik für Kinder- und Jugendmedizin, Universitätsklinikum Ulm, Ulm, Deutschland

E-Mail: martin.wabitsch@uniklinik-ulm.de 